\title{
Breaking down unitization: Is the whole greater than the sum of its parts?
}

\author{
Maria C. D’Angelo ${ }^{1}$ • Alix Noly-Gandon ${ }^{1}$ - Arber Kacollja ${ }^{1}$ - Morgan D. Barense ${ }^{1,2}$. \\ Jennifer D. Ryan ${ }^{1,2}$
}

Published online: 18 July 2017

(C) Psychonomic Society, Inc. 2017

\begin{abstract}
Memory impairments are often observed in aging. Specifically, older adults have difficulty binding together disparate elements (relational memory). We have recently shown that a cognitive strategy known as unitization can mitigate impaired relational learning in the transverse patterning task (TP) in both amnesia and healthy aging. This strategy allows items to be fused together through an interaction such that one item acts upon another. In the context of TP, unitization is comprised of three component processes: (1) fusion, (2) motion, and (3) semantic comprehension of action/consequence sequences. Here, we examine which of these components are sufficient to mitigate age-related impairments. Four groups of older adults were given either the full unitization strategy or one of the three component strategies. Each group of older adults showed impairments in memory for the relations among items under standard training instructions relative to a threshold that marks learning of a winner-take-all rule (elemental threshold). However, participants who were given either the full unitization strategy or the action/consequenceonly strategy showed improved performance, which was maintained following the 1-hour delay. Therefore, semantically rich action/consequence interactions are sufficient to mitigate age-related relational memory impairments.
\end{abstract}

Keywords Memory $\cdot$ Aging $\cdot$ Unitization $\cdot$ Fusion $\cdot$ Semantic comprehension

Jennifer D. Ryan

jryan@research.baycrest.org

1 Rotman Research Institute, Baycrest, 3560 Bathurst St., Toronto, Ontario, Canada M6A 2E1

2 Department of Psychology, University of Toronto, Toronto, Ontario, Canada
The ability to form associations among distinct elements (relational binding) is a critical component of higher order cognitive functioning, including episodic remembering, future planning, language processing, and imagination (Addis, Cheng, Roberts, \& Schacter, 2011; Cohen, 2015; Duff \& Brown-Schmidt, 2012; Konkel, Warren, Duff, Tranel, \& Cohen, 2008; Moscovitch, 2008). Relational binding is impaired in hippocampal amnesia (Ryan, Althoff, Whitlow, \& Cohen, 2000) and in aging (Ryan, Leung, Turk-Browne, \& Hasher, 2007). Relative to younger adults, older adults often exhibit intact memory for items but impaired memory for the relations among such items (Naveh-Benjamin, Guez, Kilb, \& Reedy, 2004; Old \& Naveh-Benjamin, 2008). We, and others, have shown that a strategy known as unitization can successfully mitigate age-related impairments in a relational memory task (Ahmad, Fernandes \& Hockley, 2015; Bastin et al. 2013; D'Angelo et al., 2016). Graf and Schacter (1989) first described unitization as the process whereby multiple, separate, items become represented as a single unit, either through perceiving or conceiving of a structure (e.g., semantic meaning) that would connect the disparate units. In our prior work, we have expanded on this definition by describing unitization is a cognitive strategy that circumvents relational memory difficulties by fusing distinct items into a single unit or by encouraging the fusion of items through an action whereby one item acts upon another item. In the latter case, where fusion occurs through an action/consequence sequence, the directional relations among the items can then be derived from this single unit. We have previously suggested that unitization (D'Angelo, Kacollja, Rabin, Rosenbaum, \& Ryan, 2015; Ryan, Moses, Barense, \& Rosenbaum, 2013) may consist of a number cognitive processes, including fusion (i.e., combining multiple items into a single unit), motion, and comprehension of action/consequence sequences (i.e., what happens when one item acts upon another, such as one item piercing 
another). The present study examined whether these processes are sufficient to mitigate age-related relational memory impairments.

In our previous work, relational memory and the use of unitization were investigated using the transverse patterning (TP) task (Spence, 1952), which is akin to the childhood game of rock-paper-scissors (RPS), but with semantically unrelated stimuli. In the TP task, individuals learn the relations among three items (A, B, C). Each item wins in the context of one item and loses in the context of the other item (e.g., A wins over B, B wins over C, C wins over A). Learning novel relations in TP is typically impaired in those with hippocampal amnesia (Moses, Ostreicher, Rosenbaum, \& Ryan, 2008; Rickard \& Grafman, 1998; Rickard, Verfaellie, \& Grafman, 2006; Ryan et al., 2013) and in older adults (Driscoll et al., 2003; Ostreicher, Moses, Rosenbaum, \& Ryan, 2010). Amnesic cases and older adults generally perform with lower accuracy and/or require more learning trials than control participants or younger adults. Also, amnesic cases and older adults tend to perform at or below an elemental threshold that represents learning of a winner-take-all rule (i.e., correct performance on two of the three problem sets). However, the amnesic case D.A., whose damage included the hippocampus and other regions of the medial temporal lobe bilaterally, was able to successfully perform TP (Ryan et al., 2013). D.A. was able to perform TP through the use of a strategy in which he would imagine the objects fusing together through an action/ consequence sequence in order to determine the winning object (e.g., "The star pierces the bucket"). D.A. successfully applied this unitization strategy to multiple problem sets; when he did not apply this strategy, impaired performance was observed. Subsequent research with the developmental amnesic case N.C., whose damage was restricted to the extended hippocampal system (e.g., mediodorsal nuclei of the thalamus bilaterally and volume reductions in the right fornix and both mammillary bodies), also demonstrated successful and long-lasting benefits on TP with the use of unitization (D’Angelo et al., 2015). The work with N.C. further demonstrated that self-generation of the strategy was not required for unitization to be beneficial and that the unitization strategy could be rapidly transferred to novel problem sets to support successful performance.

Like the amnesic cases D.A. and N.C., older adults often perform poorly on TP (Driscoll et al., 2003; Ostreicher et al., 2010), and they also benefit from unitization to support agerelated TP deficits (D’Angelo et al., 2016). However, older adults benefit from unitization only if they are not already experiencing early signs of cognitive decline (D'Angelo et al., 2016). In this prior work, older adults who had no subjective memory complaints and who scored within the healthy range on a neuropsychological battery, but who nonetheless failed the Montreal Cognitive Assessment (MoCA), were impaired on TP and did not benefit from unitization training. However, a group of older adults who passed the MoCA were also impaired on TP but, importantly, demonstrated significant benefits in TP performance through the use of unitization. These findings suggested that one or more cognitive processes that are required for unitization (e.g., fusion, comprehension of action/consequence sequences) may already be compromised in individuals who fail the MoCA, despite a lack of subjective cognitive complaints.

Beyond TP, unitization has been shown to be a successful strategy for mitigating age-related relational memory impairments in associative recognition tasks. For instance, Bastin et al. (2013) had younger and older adults study item-color pairings under conditions that either did (e.g., imagine the color as a feature of the item) or did not (e.g., imagine the item with a green euro bill or with a red stop sign) encourage unitization. Subsequently, participants were asked to retrieve the color associated with each item. Age-related deficits in the retrieval of the associated color were significantly reduced under unitization instructions. Likewise, age-related deficits on a yes-no associative recognition task were reduced under conditions that promoted unitization by encouraging participants to study the word pairs as a compound word rather than as two separate words (Ahmad et al., 2015), similar to prior findings from amnesic cases (Quamme, Yonelinas, \& Norman, 2007). Analyses using receiver operating characteristics have suggested that the increase in accuracy for older adults under unitized task instructions was due to an increased reliance on familiarity processes (Bastin et al., 2013; Quamme et al., 2007). Moreover, using the same paradigm as in Bastin et al. (2013), researchers have shown that engaging in unitization elicits ERP signatures consistent with the use of familiarity-based source recognition (Zheng, Li, Xiao, Ren, \& He, 2016; see also Parks \& Yonelinas, 2015).

There is one notable difference between our work with unitization in TP and studies that have examined unitization in associative recognition studies. While both paradigms require learning of novel relations among pairs of items, the TP task requires knowledge of the direction of the relation (e.g., A wins over B), whereas in the associative recognition tasks noted above, memory for directional relational information is not part of the task demands. In the associative recognition tasks, successful performance is likely facilitated by fusion alone, either by imagining one item of the pair (e.g., the color) as a feature of the other item, thereby containing both items within a single unit (Bastin et al., 2013), or by creating a new item with a novel meaning (i.e., compound word; Ahmad et al., 2015). Given the importance of directional information in TP, we predicted that fusion alone would not be sufficient to mitigate age-related impairments in relational memory. Instead, we hypothesized that comprehension of the action/ consequence sequences would be necessary and may be sufficient to support the learning of new relations. Action/ consequence sequences inherently contain information 
regarding relations among items as one item acts as an agent upon another item, thereby incorporating directionality within the fused representation.

To test these hypotheses, we compared TP in older adults who were given one of four cognitive strategies. One group was given the unitization strategy, as in our prior work (D'Angelo et al., 2016), whereby objects are fused through an action/consequence sequence, such that the directional relations among the objects are depicted. A second group was given a similar strategy that involved action/consequence sequences, but the items did not appear fused together. A third group was given a strategy to control for motion effects, in which the winning object would move and the losing object would remain static. A final group was given a fusion strategy, in which the winning and losing items were presented as separate from one another and then were shown fused together (see Fig. 1). All four groups were also given a condition of standard training with TP with which to contrast performance using a strategy. We predicted that only the strategies of unitization and action/consequence sequences would lead to significantly greater performance relative to the standard training condition. Therefore, while previous work has shown that fusion can be successfully employed as a strategy to bypass relational memory deficits, we expected that findings from the present work would highlight that the nature of the task demands is important to consider in determining which strategy can be successfully employed to support relational memory.

\section{Method}

\section{Participants}

Eighty ${ }^{1}$ healthy older adults were recruited from the Adult Volunteer Pool at the University of Toronto and the Participant Database at Baycrest Health Sciences. All participants had a passing score on the Montreal Cognitive Assessment (Nasreddine et al., 2005), as individuals who failed the MoCA did not benefit from unitization in our prior study (D'Angelo et al., 2016). Inclusion criteria included no known neurological conditions and no history of concussion. This study was approved by the University of Toronto Ethics Review Board and the Research Ethics Board at Baycrest

\footnotetext{
${ }^{1}$ Three additional participants completed the experiment but were excluded from the final data set for the following reasons. Two participants performed below an established threshold (elemental threshold, described in the caption for Fig. 2) in the training phase for their respective strategy conditions (action/ consequence and fusion). These participants were excluded because the training phases for the strategy conditions included central cues that supported learning of the problem sets. Their low performance in the training phase suggests that these participants did not understand the task demands. A third participant was excluded because their performance fell below the elemental threshold on the immediate test for RPS. As described in text, RPS was included as a baseline measure of performance.
}

Health Sciences. All participants provided written consent and received monetary compensation.

The 80 participants were randomly assigned to one of four strategy groups, which differed based on the strategy given during training (see below). Mean age did not differ between groups, nor did years of education, MoCA, or ERVT score $(F$ $<1$ for all tests). (For means and ranges, see Table 1.)

\section{Apparatus}

As in our prior work (D'Angelo et al., 2015; D'Angelo et al., 2016; Ryan et al., 2013), the experiment was programmed using E-Prime (V.1.1). Participants tested at the University of Toronto completed the experiment on a Dell laptop computer, while participants tested at Baycrest Health Sciences completed the experiment on a Dell desktop computer connected to a 19-inch monitor.

\section{Stimuli and procedures}

Unique sets of three stimuli were used for each of the three conditions: TP with standard training (standard), TP with a strategy during training (strategy), and RPS (see Fig. 1a). The conditions differed in terms of how training was conducted and in the extent to which the relations were semantically rich. The standard and strategy conditions contained unknown abstract objects with unknown relations. These objects were previously used in Moses, Ostreicher, and Ryan (2010) and were used here to reduce the likelihood that performance would be at ceiling (D'Angelo et al., 2016), as our prior work has generally found lower TP performance with unknown objects relative to geometric shapes (D'Angelo et al., 2015; Ostreicher et al., 2010; Ryan et al., 2013). The RPS stimuli depicted the hand game where rock crushes scissors, scissors cut paper, and paper covers rock. Unlike the two conditions with abstract objects, the stimuli and relations in the RPS condition are semantically meaningful, and performance on this condition is generally intact in healthy older adults (D'Angelo et al., 2016; Ostreicher et al., 2010) and in amnesic cases (D'Angelo et al., 2015; Moses et al., 2008; Ryan et al., 2013).

In all conditions, participants were trained on the relations $\mathrm{A}+\mathrm{B}-, \mathrm{B}+\mathrm{C}-, \mathrm{C}+\mathrm{A}-$. Participants in all groups first completed training and an immediate test in the standard condition, followed by training and an immediate test in the strategy condition. To minimize the influence of working memory/online maintenance strategies on test performance (D'Angelo et al., 2015; see Ryan et al., 2013), participants were tested again on the relations in the standard and strategy conditions following an hour delay. After the delayed tests, participants were trained and given an immediate test on the RPS condition. The standard condition was included as a baseline measure with which to compare groups when learning novel relations, while the RPS condition was included as a baseline measure to compare the groups on 
A Stimuli
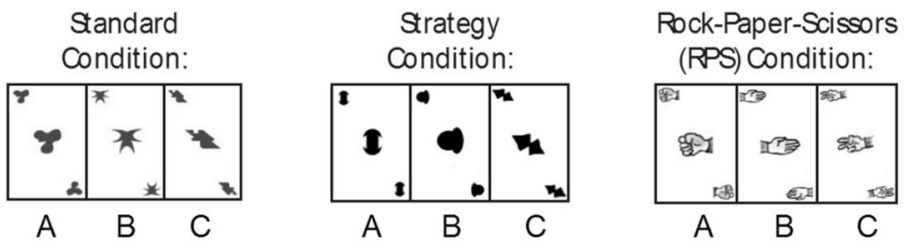

(RPS) Condition:

B Animations

For illustration, four still images shown from the A+B-pair for each Strategy condition.

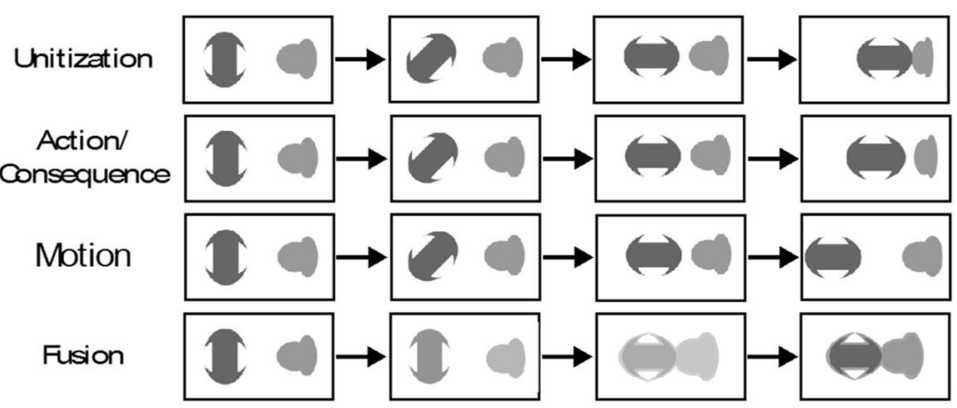

C Central Strategy Reminder Cues

Central strategy reminder cues for each strategy:

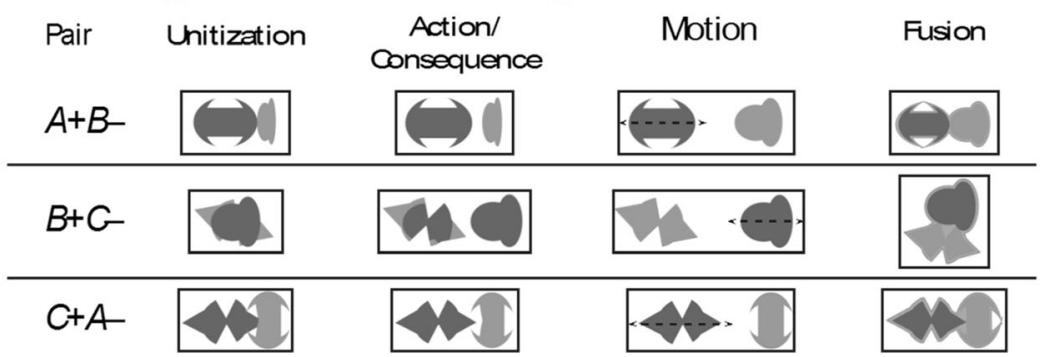

Fig. 1 Stimuli and training procedures for the standard, strategy, and rock-paper-scissors (RPS) conditions. a The stimuli used in the standard and the strategy conditions were three abstract objects that were preexperimentally unknown to the participants. The RPS stimuli depicted the three gestures used in the hand game. In all cases, the rules underlying

TP with semantically rich relations. All participants were provided with the same testing order (standard condition first, RPS last). This allowed us to examine performance on the standard condition without any carryover bias in strategy use that may have occurred if the strategy condition had been presented first.

Table 1 Mean (and ranges) of participant ages, education, MoCA scores, and score on the Extended Range Vocabulary Test (ERVT) as a function of group

\begin{tabular}{lllll}
\hline Group & Age & Education & MoCA (/30) & ERVT (/48) \\
\hline Unitization & 71.4 & 16.9 & 28.1 & 33.6 \\
& $(65-88)$ & $(10-26)$ & $(26-30)$ & $(17.75-48)$ \\
Action/consequence & 71.8 & 17.3 & 27.7 & 34.7 \\
& $(62-81)$ & $(11-30)$ & $(26-30)$ & $(15.75-47)$ \\
Movement & 71.5 & 16.9 & 27.5 & 32.9 \\
& $(61-86)$ & $(12-23)$ & $(26-30)$ & $(5.5-48)$ \\
Fusion & 72.2 & 16.6 & 28.1 & 36.0 \\
& $(62-82)$ & $(12-22)$ & $(26-30)$ & $(16.75-45.75)$ \\
\hline
\end{tabular}

the TP task applied $(\mathrm{A}+\mathrm{B}-, \mathrm{B}+\mathrm{C}-, \mathrm{C}+\mathrm{A}-;++=$ rewarded/correct choice, = unrewarded/incorrect choice). $\mathbf{b}$ Example stills from the flash animations for the $\mathrm{A}+\mathrm{B}-$ pair shown as part of the strategy TP training conditions. $\mathbf{c}$ The three central reminder cues corresponding to the three pairs are shown for each of the four strategy conditions

As well, our prior work (Ostreicher et al., 2010) has shown that providing RPS first enhances subsequent performance on the standard condition, presumably due to the use of a known relational framework (RPS) onto which new learning (the relations in the standard condition) can be integrated. Providing RPS before either the standard or strategy conditions would have made it difficult to understand the nature of baseline performance and/or whether any component(s) of unitization provide a benefit to performance.

Standard training Training procedures are depicted in Fig. 2 and were identical to our prior work (D'Angelo et al., 2015; D’Angelo et al., 2016; Ryan et al., 2013). Participants were not given information about the relations among the stimuli and were required to learn the relations through trial and error. On every trial, participants were shown two stimuli and were instructed to select one of the objects as a winner. Participants responded using the "Q" and "P" keys on the keyboard to 


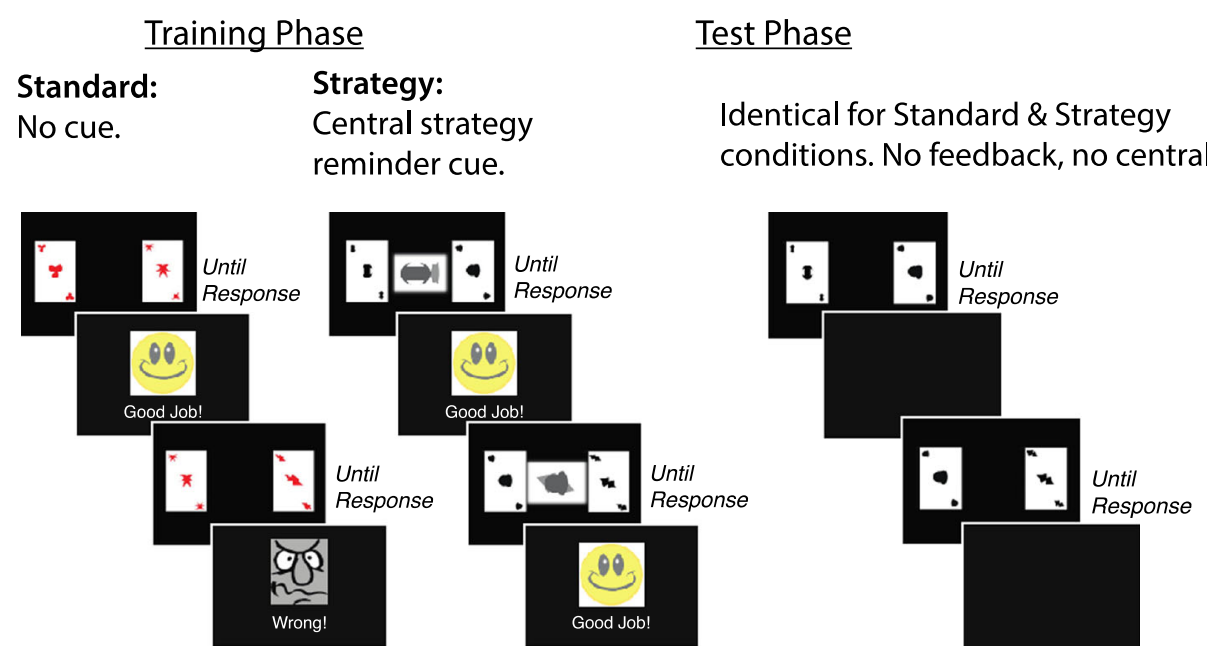

Fig. 2 The training phase for the standard condition depicted two stimuli, from which the participant had to select the "winner." Responses were self-paced and feedback was provided after every trial. Training for the RPS condition followed the same procedures as the standard condition. Strategy training proceeded in the same manner as the standard training with the following exceptions: (1) participants were first shown the animations, and (2) a central still image from the animation was included to

select the left and right stimulus, respectively. The winning stimulus was presented equally often on the left versus right sides of the monitor. Feedback was presented on every trial. On correct trials participants saw a happy-face cartoon, with the caption "Good Job!". On incorrect trials participants saw an angry-face cartoon, with the caption "Wrong!". Mean accuracy was presented at the end of each block of trials.

As in our prior work (D'Angelo et al., 2016; Ryan et al., 2013), training unfolded over five stages. In the first stage, participants completed a block of 10 trials of the first pair of objects $(\mathrm{AB} \times 10)$, followed by a block of 10 trials of the second pair of objects ( $\mathrm{BC} \times 10)$, and, last, a block of 10 trials of the third pair of objects $(\mathrm{CA} \times 10)$. In the second stage, participants completed a block of 15 trials in which each of the pairs were presented in consecutive order, for five presentations $([\mathrm{AB}, \mathrm{BC}, \mathrm{CA}] \times 5)$. In the third stage, participants completed three blocks of nine trials. In each block the three pairs were presented consecutively for three presentations $([\mathrm{AB}, \mathrm{BC}, \mathrm{CA}] \times 3)$. In the fourth stage, participants completed a block of 27 trials in which each pair was presented in consecutive order for nine presentations ([AB, $\mathrm{BC}, \mathrm{CA}] \times 9$ ). Last, in the fifth stage, participants completed two blocks of 54 trials in which each pair was presented 18 times in a pseudorandom order. If accuracy for a block of trials was less than $50 \%$, the block was repeated. Participants completed a minimum of 207 trials during training.

Strategy training Training in the strategy conditions was identical to training in the standard condition with the following exceptions. Animations were created for each of the four strategies. Three animations were created for each strategy, one for each of three pairs of objects (A-B, B-C, C-A; see Fig. 1b). serve as a reminder for which stimulus was the "winner" as in the animations. During the test phase, all conditions (standard, strategy, RPS) followed the same procedures; two stimuli were presented, one on each side of the screen, and participants were asked to select the "winner." Responses were self-paced, and feedback was not provided. (Color figure online)

These animations were created using Adobe Flash and differed in the extent to which the objects moved and interacted with one another. As the stimuli used were unknown prior to the experiment, the winning object was always shown in dark gray and the losing object in light gray. The difference in shade was included so that the experimenter could describe the videos without using object labels. The animations were presented to the participants prior to beginning the training phase. The instructions given differed based on the strategy given and are described below. The full sets of instructions for each strategy are presented in the Appendix.

Unitization strategy The unitization animations were identical to those used in our prior work (D'Angelo et al., 2015; Ryan et al., 2013). Each animation depicted two items interacting with one another such that a winner was made clear. The first animation depicted object A squishing object $\mathrm{B}$. The second animation depicted object $\mathrm{B}$ covering object $\mathrm{C}$. And the third animation depicted object $\mathrm{C}$ stabbing object $\mathrm{A}$. The animations always ended with the two items touching in a way that encouraged fusion.

Participants in the unitization strategy group were first told the following: "We think that it may help to imagine the objects interacting. These movies show the objects in two colors, interacting so that you can tell which one should be the winner." As each animation was presented, the experimenter verbally described the animation and asked the participant to confirm that they understood which object was the winner in each pair. For example, for pair $\mathrm{AB}$, the experimenter would tell the participant, "In this video, the dark object squishes the light object, 
making the dark object the winner. Please indicate which object is the winner."

Action/consequence strategy The action/consequence animations were identical to the unitization animations with the exception that the items never touched. As in the unitization condition, each animation in the action/consequence condition depicted the winning object moving, and as a result of the action, the losing object would change. For example, the first animation depicted object $\mathrm{A}$ moving toward object $\mathrm{B}$, with object $\mathrm{B}$ becoming squished as a result. Critically, the items never touched and the animations never ended with the two items in a fused state.

In the action/consequence strategy group, participants were first told, "We think that it may help to imagine one object moving and changing the other object. These movies show the objects in two colors, with one object moving and changing the other object so that you can tell which one is the winner." As in the other conditions, the experimenter verbally described the animations and asked the participant to confirm that they understood which object was the winner in each pair. For example, for pair $\mathrm{AB}$, the experimenter would tell the participant, "In this video, the dark object moves towards the light object and the light object gets squished, and so the dark object is the winner. Please indicate which object is the winner."

Motion strategy The motion animations were similar to the action/consequence animations in that the winning object would move, but critically, the losing object would not change. The winning objects initially followed similar motion paths as in the action/consequence animations but would retrace their path part way through. This was done so that the two objects would not touch.

In the motion strategy group, participants were first told, "We think that it may help to imagine one of the items moving. These movies show the objects in two colors, with one object moving so that you can tell which one is the winner." Again, the experimenter verbally described the animations and asked the participant to confirm that they understood which object was the winner in each pair. For example, for pair $\mathrm{AB}$, the experimenter would tell the participant, "In this video, the dark object moves left and right, and so it is the winner. Please indicate which object is the winner."

Fusion strategy The fusion animations each depicted two items that were initially presented separately, and in a subsequent frame, the items were presented as a single fused object; no continuous motion was present. The items did not interact with one another, other than through fusion. The darker shading depicted the winning item within the fused object.

In the fusion group, participants were first told the following: "We think that it may help to imagine the objects coming together into one. These movies will show the objects in two colors fusing together into one object so that you can tell which one should be the winner." Once again, as each animation was presented, the experimenter verbally described the animation and asked the participant to confirm that they understood which object was the winner in each pair. For example, for pair $\mathrm{AB}$, the experimenter would tell the participant, "In this video, the two objects come together to form a new object, and this feature of the object is the winning part. Please indicate which part is the winner."

Following presentation of the animations, participants completed the training phase of the experiment. The training phase was identical to the standard training with the following exception. Participants were reminded of the animations through the presentation of a central cue. The cues were drawn from the last frames of the animations and were always paired with the appropriate stimulus (i.e., if the present trial contained pair $\mathrm{AB}$, the central cue was drawn from the animation for pair $\mathrm{AB})$. Feedback was provided following each trial, as in standard training.

Test phase The immediate and delay test phases were identical for the standard condition and for each of the strategy conditions (unitization, action/consequence, fusion, motion). Each pair of items was presented four times during the test phase, for a total of 12 trials. Participants were not given feedback during the test phase. Central cues were not presented in the test phase for the strategy condition.

RPS Training and test procedures for RPS followed the standard training procedures as noted above, with one exception. Participants were queried as to whether they were familiar with the RPS game and the hand gestures, and to ensure that they understood the direction of the relations within RPS. As in the standard training, no animations of the sets of RPS stimuli were provided, and no central cue was provided. The test phase proceeded in the same manner as for the standard and each of the strategy conditions.

Postexperimental questionnaires Following each test phase, participants were given questionnaires to assess their awareness of the relations among the items. These questionnaires were identical to our prior work (Ostreicher et al., 2010).

\section{Analysis}

Performance was compared for the standard and strategy TP conditions separately for each experimental phase (training/ immediate test/delay test) using mixed-effects ANOVAs. Each ANOVA included TP Training as a within-subjects factor with two levels (standard/strategy) and strategy group as a between-subjects factor with four levels (unitization/actionconsequence/motion/fusion). 
Given that RPS was included in the experimental design to serve as a baseline measure to ensure that all groups could perform TP with semantically rich relations, separate ANOVAs were run for RPS performance for the training and immediate test phases. Accuracy for RPS was analyzed using a one-way ANOVA, with strategy group (unitization/actionconsequence/motion/fusion) included as a between-subjects factor.

\section{Results}

\section{Standard versus strategy TP conditions}

Training phase Mean accuracy in the training phase as a function of strategy group and TP training condition are listed in Table 2. The ANOVA of accuracy in the training phase revealed higher accuracy with strategy TP training $(M=$ $0.97, S E=0.00)$ than standard TP training $(M=0.60, S E=$ $0.01), F(1,76)=907.01, p<.001, \eta_{\mathrm{p}}{ }^{2}=0.92$. The main effect of strategy group and its interaction with TP Training were not significant $(F<2, p>.14$ for both).

Immediate test phase Mean accuracy in the immediate test phase as a function of strategy group and TP training condition are shown in top row of Fig. 3. The analysis revealed higher accuracy in the strategy TP training conditions $(M=$ $0.70, S E=0.03)$ than in the standard TP training condition $(M$ $=0.57, S E=0.02), F(1,76)=8.70, p=.004, \eta_{\mathrm{p}}{ }^{2}=0.10$. The main effect of the strategy group was marginally significant, $F(3,76)=2.45, p=.070, \eta_{\mathrm{p}}{ }^{2}=0.09$, reflecting the pattern of higher overall accuracy in the action/consequence condition $(M=0.69, S E=0.04)$ and lower overall accuracy in the motion condition $(M=0.55, S E=0.03)$, relative to the unitization $(M=0.64, S E=0.04)$ and fusion $(M=0.61, S E=0.04)$ conditions. The interaction between the two factors was not significant, $F(3,76)=1.89, p=.139, \eta_{\mathrm{p}}{ }^{2}=0.07$.

Delay test phase Mean accuracy in the delay test phase as a function of strategy group and TP Training condition are

Table 2 Mean accuracy ( $S E)$ on standard and strategy conditions at training as a function of experimental strategy group

\begin{tabular}{cccccc}
\hline & \multicolumn{4}{c}{ Experimental strategy group } \\
\cline { 3 - 6 } & & Fusion & Motion & $\begin{array}{l}\text { Action/ } \\
\text { consequence }\end{array}$ & Unitization \\
\hline TP & Standard & $\begin{array}{c}0.63 \\
(0.03)\end{array}$ & $\begin{array}{c}0.60 \\
(0.02)\end{array}$ & $0.58(0.02)$ & $0.57(0.02)$ \\
training & Strategy & $\begin{array}{c}0.98 \\
(0.01)\end{array}$ & $\begin{array}{c}0.96 \\
(0.02)\end{array}$ & $0.97(0.00)$ & $0.99(0.00)$ \\
& & \multicolumn{5}{c}{$(0.0)$} & \\
\hline
\end{tabular}

shown in bottom row of Fig. 3. The analysis revealed higher accuracy in the strategy TP training condition $(M=0.68, S E=$ $0.03)$ than in the standard TP training condition $(M=0.57, S E$ $=0.02), F(1,76)=10.96, p=.001, \eta_{\mathrm{p}}{ }^{2}=0.13$. The main effect of the strategy group was not significant, $F(3,76)=2.32, p=$ $.082, \eta_{\mathrm{p}}{ }^{2}=0.08$.

Importantly, the interaction between TP training condition and strategy group was significant, $F(3,76)=3.28, p=.025$, $\eta_{\mathrm{p}}{ }^{2}=0.11$. To investigate the nature of this interaction, pairedsamples $t$ tests were employed to contrast performance in the standard versus strategy TP conditions, separately for each strategy group. These paired-samples $t$ tests confirmed that the interaction was driven by a difference in accuracy on the strategy versus standard TP conditions in the unitization and action/consequence groups that was not observed in the motion and fusion groups. Specifically, we replicated our previous finding of higher accuracy with unitization TP training ( $M$ $=0.78, S E=0.04)$ relative to standard TP training $(M=0.57$, $S E=0.05), t(19)=2.88, p=.01$. We observed a similar pattern with action/consequence TP training $(M=0.78, S E=0.06)$, where accuracy was higher relative to standard TP training $(M$ $=0.56, S E=0.05), t(19)=2.95, p=.008$. Performance on the strategy TP Training did not differ between the unitization $(M$ $=0.78, S E=0.05)$ and action/consequence groups $(M=0.78$, $S E=0.06), t(38)=0, p=1$. These results suggest that the comprehension of action/consequence sequences is a sufficient cognitive component of unitization in mitigating agerelated impairments in TP. In contrast to the differences observed in the unitization and action/consequence groups, performance was not significantly higher in the strategy TP condition relative to the standard TP condition in the motion and fusion groups, $t(19)=0.36, p=.723$, and $t(19)=-0.15, p=$ .885 , respectively, suggesting that these strategies did not mitigate age-related impairments in TP.

To determine whether the unitization and action/ consequence strategies mitigated impaired performance, performance on the strategy TP training was compared to the elemental learning threshold (accuracy $=0.67$ ). The elemental threshold reflects the maximum score achievable if an elemental learning rule is incorrectly applied to a TP task (e.g., a winner-takes-all rule). Use of an elemental learning rule will result in correctly learning only two thirds $(67 \%)$ of the relations. Individuals who perform at or below this criterion are considered to have impaired relational learning (D'Angelo et al., 2015; Rickard \& Grafman, 1998; Rickard et al., 2006; Ryan et al., 2013). Mean accuracy on the delay test for each strategy TP training condition was compared to the elemental threshold using one-tailed one-sample $t$ tests. These $t$ tests confirmed that mean accuracy was significantly higher than the elemental threshold in the unitization, $t(19)=2.05, p=$ .027 , and action/consequence, $t(19)=1.81, p=.043$, conditions, but not in the motion, $t(19)=-2.10, p=.975$, and fusion, $t(19)=-1.92, p=.965$, conditions. 


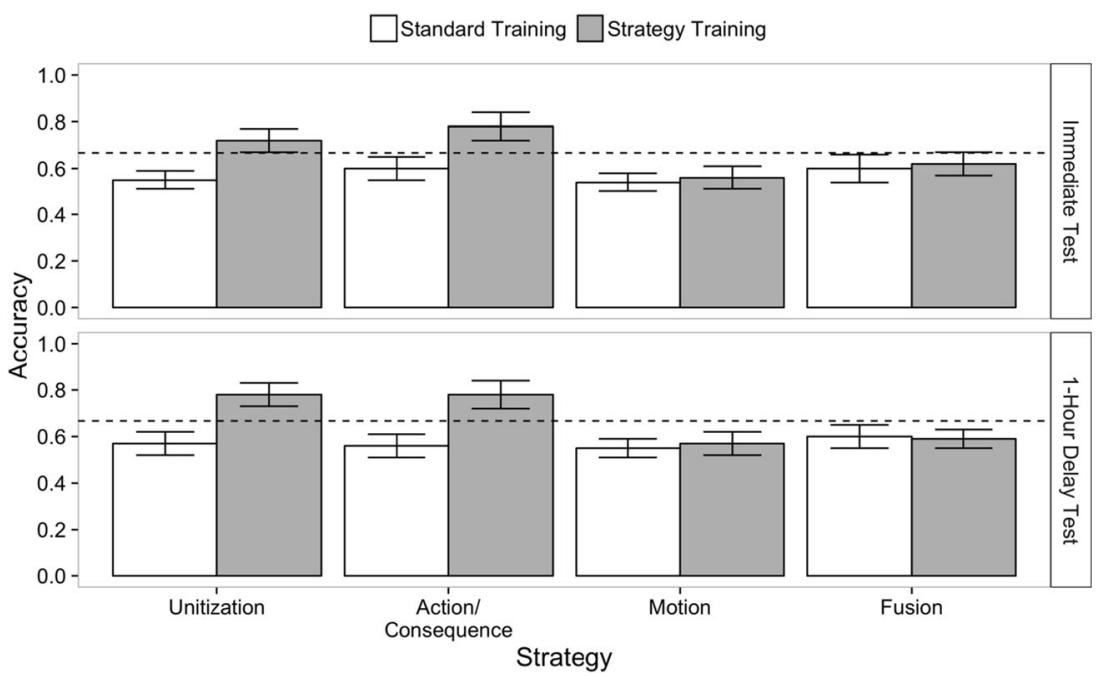

Fig. 3 Mean accuracy as a function of strategy type (unitization, action/ consequence, motion, and fusion) and TP training condition (standard vs. strategy) for both test phases. Error bars represent standard error of the

mean. Dashed line represents the elemental learning threshold (0.67). (See text for additional details.)

\section{RPS}

Mean accuracy in the training and test phases for the RPS condition as a function of strategy group are listed in Table 3. Performance on RPS was high in all groups and accuracy did not differ across groups at training, $F(3,66)=$ $1.12, p=.348$, nor did it differ across groups at test, $F(3,66)=$ $1.66, p=.185$.

\section{Discussion}

Unitization is a strategy that can support performance on tasks that require learning the relations among items, and it can support transfer of learning to new problem sets (D'Angelo et al., 2015). We have defined unitized representations as those consisting of items that are fused through an action and are integrated with existing information within semantic memory. Relations among the distinct items may be subsequently derived from these fused representations (D'Angelo et al., 2015; D’Angelo et al., 2016; Ryan et al., 2013). Here, we examined which of the component cognitive processes that

Table 3 Mean accuracy ( $S E$ ) on RPS condition at training and immediate test as a function of experimental strategy group

\begin{tabular}{cccccc}
\hline & & \multicolumn{3}{l}{ Experimental strategy group } \\
\cline { 3 - 6 } & & Fusion & Motion & $\begin{array}{l}\text { Action/ } \\
\text { consequence }\end{array}$ & Unitization \\
\hline RPS & Training & $\begin{array}{c}0.98 \\
(0.00)\end{array}$ & $\begin{array}{c}0.97 \\
(0.01)\end{array}$ & $0.98(0.00)$ & $0.98(0.00)$ \\
Phase & $\begin{array}{c}\text { Immediate } \\
\text { test }\end{array}$ & $\begin{array}{c}0.96 \\
(0.01)\end{array}$ & $\begin{array}{c}0.98 \\
(0.01)\end{array}$ & $0.99(0.01)$ & $0.99(0.01)$ \\
& & & & & \\
\hline
\end{tabular}

compose unitization are sufficient to support task performance on TP, a task for which successful performance requires that the directionality of the relations must be learned (i.e., A wins over B, B wins over C, C wins over A). In four groups of older adults, we contrasted performance under standard training conditions against one of four strategy conditions (unitization, action/consequence sequences, fusion, and motion) and found that knowledge of action/consequence sequences may be a critical cognitive component of the unitization strategy that drives successful performance here on TP.

During training, the use of any strategy resulted in higher performance compared to the standard condition in which no strategy was provided. This is perhaps not surprising as, in the strategy conditions, the winning object was revealed in the central cue that remained on the screen for the duration of each of the training trials. During the test phases, these central cues were removed, and performance remained higher for the strategy conditions, overall, compared to the standard condition. At immediate test, no significant difference emerged among the strategy conditions, although accuracy was numerically higher in the unitization and the action/consequence conditions. At the 1-hour delay test, a significant interaction emerged such that accuracy was significantly higher only for the groups of older adults who were provided with either the full unitization strategy, or the action/consequence strategy, compared to the standard condition. Moreover, at the 1-hour delay, it was only the unitization and action/consequence strategy groups who exhibited performance above the elemental threshold, which represents learning two out of three relations and is typically used as the threshold for which relational learning is considered to have occurred (D'Angelo et al., 2015; Rickard \& Grafman, 1998; Rickard et al., 2006). Neither the motion nor the fusion strategy conferred any advantage over standard training, and neither strategy supported 
performance above the elemental threshold at the immediate and 1-hour delay tests. Thus, in a task that required learning the directionality of the relations among items, fusion was neither necessary nor sufficient to support performance, whereas the use of action/consequence sequences was sufficient, and perhaps even necessary, for high accuracy to occur on TP.

Participants in the four strategy groups (unitization, action/ consequence, motion, fusion) performed similarly, and near/at ceiling, on RPS, suggesting that there were no baseline differences across groups. High RPS performance for each of the strategy groups suggests that each of the participant groups understood the task demands, and had intact access to, and use of, prior semantic knowledge. Participants in each of the strategy groups also performed similarly when they received standard TP training, with performance in all groups below the elemental threshold on the standard condition. This agerelated impairment in relational memory, as expressed by performance on the standard condition, is consistent with previous findings of age-related impairments on TP (Ostreicher et al., 2010), even in a group of nominally healthy older adults who passed the MoCA and performed within the normal range on a variety of neuropsychological tests (D'Angelo et al., 2016). The present findings add to our prior work with older adults (D'Angelo et al., 2016) and amnesic case N.C. (D'Angelo et al., 2015), demonstrating that unitization is a viable strategy to bypass relational memory deficits and that self-generation of the strategy is not required for successful performance to occur. The present work extends these prior findings by demonstrating that the use of action/consequence knowledge was sufficient to support performance on TP, at accuracy levels akin to what was observed when the full unitization strategy was used. Given that participants in each of the strategy groups were matched for age and education, and that there were no group differences in performance on standard TP and baseline RPS, it is unlikely that the high performance by the older adults in the unitization or the action/ consequence strategy conditions was due to factors other than the use of the respective strategies.

Previous research has indicated that errorless learning may be a method by which amnesic cases can circumvent declining memory function (Glisky et al., 1986). The present findings suggest that errorless learning is not sufficient to support performance on TP, consistent with our prior work with older adults (D'Angelo et al., 2016) and amnesic cases (D'Angelo et al., 2015; Ryan et al., 2013). Near perfect training was observed in each of the strategy conditions, due to the use of the central cue. If errorless learning were critical for the success of unitization, then we would have expected test performance to be high in all of the strategy conditions, which was not the case. High accuracy that was observed during training was not maintained in the test phases for either the motion or the fusion conditions. By contrast, test performance for the unitization and action/consequence conditions was above the elemental threshold at the 1-hour delay. Thus, the success of either the unitization or action/consequence strategies in learning the directionality of the relations was likely not due to the errorless learning format that occurred during training. Nonetheless, as noted in our previous work (D'Angelo et al., 2015), errorless learning may have contributed to the strengthening of the representations that were developed during training (Glisky et al., 1986). In this case, while representations may have been formed and strengthened in each of the strategy conditions, only the representations from the unitization and the action/consequence conditions would have contained information regarding the directionality of the relations that was necessary to support performance on TP.

There are cognitive processes involved in unitization, beyond those that were investigated here, that may aid in the learning of relations. That is, although comprehension of action/consequence sequences was sufficient for successful performance, we have previously noted that other processes such as imagery, access to semantic memory, and online maintenance of information (or elaborative processing more generally), may contribute to the broad success of unitization strategies (D'Angelo et al., 2015; D'Angelo et al., 2016; Ryan et al., 2013). In particular, there is evidence to suggest that imagery may be a key component to successful relational learning and retrieval (Bower, 1970; Cermak, 1975; McGee, 1980) and to the successful performance observed in associative recognition tasks in which the unitization instructions required participants to imagine one item as a feature of another, or to imagine a new object made of the individual components. In Bastin et al. (2013), performance was higher with the use of a unitization strategy, but only for those trials in which the unitized association was judged to be easy to imagine. Here, participants likely engaged in imagery with the action/consequence strategy. In order to control for any advantages due to the explicit presentation of fusion, the objects in the action/consequence strategy did not touch; therefore, participants were left to infer the action of one object onto another with the resulting consequence. Thus, we would predict that participants who have declining imagery abilities, particularly for interactions that are not already part of semantic memory, may not benefit from unitization and/or action/consequence strategies in the learning of directional relations.

In addition to imagery, we expect that intact maintenance (working memory), and/or the ability to engage in elaborative processing, may be important component processes that enable the ongoing formation and strengthening of unitized representations within an errorless learning format. Our previous observations of a lack of a unitization benefit in amnesic cases K.C. and R.F.R. may have reflected lower online maintenance/working memory abilities in K.C. and R.F.R. as compared to D.A. and N.C. for whom unitization was successful (D’Angelo et al., 2015; Ryan et al., 2013). Further 
work remains to test these other component processes of unitization and/or to test the effectiveness of the action/ consequence strategy in populations with declining imagery, online maintenance/working memory, and/or access or use of semantic memory. We would predict, based on our prior work with older adults who failed the MoCA (D'Angelo et al., 2016), that deficits in any/all of these component processes may result in a lack of benefit for the action/consequence strategy.

While it has been proposed that familiarity may be a key component process in the successful use of unitization (Bader, Mecklinger, Hoppstadter, \& Meyer, 2010; Bastin et al., 2013;Delhaye \& Bastin, 2016), the findings here suggest that is the content within the unitized representation that critically determines task success. That is, the unitized representations form the bedrock upon which cognitive processes, like familiarity, are based. Prior work has shown that fusion strategies allow for the increased reliance on familiarity presumably due to the development of a single unitized representation upon which familiarity can act (Bastin et al., 2013; Yonelinas, 1997), yet here, the fusion strategy did not support performance above levels observed in the standard condition, and performance was not above the elemental threshold. It remains a possibility that our version of a fusion strategy did not elicit the same reliance on familiarity as in other studies, either due to the difference in stimuli used (novel and perceptually distinct objects in the present task versus known words or single objects in previous studies), or due to nuanced differences in the nature of the task demands (respond based on a directional relationship between items as in the present task vs. remember a pairing of stimuli as in previous studies). However, we suggest that successful performance was not observed in the present work with the fusion strategy because the fused representations did not contain directional relational information as required by the task. Also, although measures of recollection/familiarity were not a part of the present work, we believe it is unlikely that differences in the engagement of familiarity emerged across the strategy conditions, although such possibilities remain to be tested. We suggest that in TP, or in any task in which directional relational learning is required, familiarity - in and of itself - will be insufficient to support performance. This position is in line with other work that demonstrates that the content of representations, and not the processes engaged (i.e., recollection/familiarity), determines whether task performance will be successful (Watson, Wilding \& Graham, 2012).

The present work provides further evidence demonstrating the successful use of unitization to circumvent deficits in relational learning and memory in older adults (Ahmad et al., 2015; Bastin et al., 2013; Kan et al., 2011) as has been done with amnesic cases (D'Angelo et al., 2015; Ryan et al., 2013; Quamme et al., 2007). We have added to this growing field by demonstrating which of the cognitive components that compose unitization are sufficient to support successful performance when the directionality of the relations must be learned. The present findings highlight the fact that although multiple research groups, including ours, have used the term unitization to refer to the collective set of cognitive processes whereby multiple items and the relations among them are stored as single unit, studies have varied with respect to the nature of the relations that are to be learned. As a result, different components of the unitization strategy likely become necessary and/or sufficient to support performance. In associative recognition tasks, the only requirement is to learn that two items are to be associated; therefore, multiple items may be blended together into a single unit through fusion alone. Use or knowledge of action/consequence sequences may be unnecessary, although this remains to be tested given that some associative recognition tasks that invoke unitization ask participants to imagine items interacting (Bastin et al., 2013), which may result in the unintentional use of imagery of action/consequence sequences by the participants during learning. Here, the fusion strategy did not contain information regarding the directionality of the relations, and was not sufficient to support TP performance. Instead, we suggest that the action/consequence strategy allowed for the items to become fused into a single, unitized, representation, and incorporated information regarding the directionality of the relations into that fused representation such that the relations could be subsequently derived (e.g., the bucket has been pierced by the star, therefore the star wins over the bucket).

Thus, as conceptualized here, the action/consequence strategy likely (and perhaps obligatorily) invokes other component strategies, including motion, and imagery of the items becoming fused into a single item through the action sequence. The present work attempted to control for any benefit of motion and fusion by examining performance of these strategies separately, and neither successfully supported TP performance, suggesting that the action/consequence strategy provided the requisite information to support performance. However, an action/consequence strategy that includes elements of motion and fusion may enable the development of a richly detailed, unitized, representation that includes information regarding the directionality of relations.

However, if we consider that it is the learning of these directional relations that is critical for TP performance here, then fusion, motion, and even unitization in the broader sense, may not be necessary to support performance on TP. Rather, the knowledge regarding the directionality of relations that is learned via the action/consequence sequence may successfully support performance, without the requirement for fusion, or the development of a single, unitized, representation. Indeed, there is considerable evidence that propositional knowledge, including cause-effect knowledge that is depicted within the action/consequence condition wherein the directionality of the relations is conveyed, facilitates new learning and supports 
memory (Ericsson \& Kintsch, 1995; Kintsch, 1988). We have shown that prior propositional knowledge supported new learning in older adults on TP (Moses et al., 2010); specifically, exposure to the preexperimentally known rock-paperscissors version of TP facilitated the learning of relations among a novel set of stimuli. However, amnesic cases could not use such prior knowledge regarding the directionality of relations and apply that knowledge to a novel problem set (Moses et al., 2008). Combining these prior findings with other research suggesting that amnesic cases typically show larger memory impairments for relations than for items (see Eichenbaum \& Cohen, 2001, for review), we suggest that the action/consequence and the unitization strategies, as applied here and in our in prior work, not only allowed for the directionality of relations to be maintained but allowed those relations to be maintained in a fused representation, thereby promoting successful TP performance in the amnesic cases D.A. (Ryan et al., 2013) and N.C. (D'Angelo et al., 2015) as well as in aging (D'Angelo et al., 2016). The present work, then, extends classic research regarding the strategies that enhance memory performance (e.g., Paivio, 1991) to specify the constraints by which such strategies must operate in order to support performance in special cases, such as in amnesia or in aging.

It is important to note that other strategies, beyond those that were studied in the present work, may have produced similar effects to what was observed here with the action/ consequence strategy, provided that the strategy allowed for the directionality of the relations to be maintained in a lasting, unified, representation. Consequently, we suggest that the nature of the task demands - here, the nature of the relations to be learned (i.e., associative vs. directional)-determined the component process of unitization that was required to support successful performance. Numerous, distinct, strategies may be sufficient to support performance on any task, provided that the strategy provides a means to learn and maintain the informational content (e.g., directionality of relations) and representational format (e.g., single, fused, representation) necessary to support successful performance within a given individual.

In addition to matching strategies to task demands, it is important to understand the cognitive and neural profile of an individual to further refine selection of appropriate strategies to support task performance. As noted earlier, unitization was not successfully used by the amnesic cases K.C. and R.F.R. in our prior work, despite extensive training (Ryan et al., 2013). Nor was unitization successful for a subgroup of older adults who did not pass the MoCA (D'Angelo et al., 2016). A unitization strategy that includes the use of action/ consequence sequences can support the directional learning of relations, thereby matching the strategy to the task demands. However, despite this strategy-task match, unitization was unsuccessful for the amnesic cases K.C., R.F.R., and older adults who failed the MoCA, presumably due to declines in the critical cognitive and neural components that are invoked by unitization. Therefore, to successfully bypass relational memory deficits in any individual, the cognitive strategy must be matched to the task demands, and must also be consistent with an individual's cognitive and neural profile (i.e., personalized medicine).

Acknowledgements This research was supported in part by a postdoctoral fellowship from the Canadian Institutes of Health Research (CIHR) awarded to M.C.D. as well as support from the Ontario Ministry of Health and Long Term Care through the Ontario Research Coalition of Research Institutes/Centres on Health \& Aging (ORC) to M.C.D. This work was also supported by CIHR Operating Grants awarded to J.D.R. (No. MOP126003) and M.D.B. (No. MOP-115148), and by Tier II Canada Research Chair Awards awarded to J.D.R. and M.D.B. The authors state that they do not have any conflicts of interest to declare.

\section{Appendix}

Instructions for each condition and each animation

\section{Unitization strategy}

- Overall instructions: "We think that it may help to imagine the objects interacting. These movies show the objects in two colours, interacting so that you can tell which one should be the winner."

- Pair AB: "For example, in this video, the dark object squishes the light object, making the dark object the winner."

- Pair BC: "In this video, the dark object covers the light object, making the dark object the winner."

- Pair CA: "And finally, in this video, the dark object stabs the light object, making the dark object the winner"

\section{Action/consequence strategy}

- Overall instructions: "We think that it may help to imagine one object moving and changing the other object. These movies show the objects in two colours, with one object moving and changing the other object so that you can tell which one is the winner."

- Pair AB: "For example, in this video, the dark object moves towards the light object and the light object gets squished, and so the dark object is the winner."

- Pair BC: "In this video, the dark object moves towards the light object and the light object gets covered by the other object's shadow, and so the dark object is the winner."

- Pair CA: "And finally, in this video, the dark object moves towards the light object, and the light object gets stabbed, and so the dark object is the winner." 


\section{Motion strategy}

- Overall instructions: "We think that it may help to imagine one of the items moving. These movies show the objects in two colours, with one object moving so that you can tell which one is the winner."

- Pair AB: "For example, in this video, the dark object moves left and right, and so it is the winner."

- Pair BC: "In this video, the dark object moves left and right, and so it is the winner."

- Pair CA: "And finally, in this video, the dark object moves left and right, and so it is the winner."

\section{Fusion strategy}

- Overall instructions: "We think that it may help to imagine the objects coming together into one. These movies will show the objects in two colors fusing together into one object so that you can tell which one should be the winner."

- Pair AB: "For example, in this video, the two objects come together to form a new object, and this feature of the object is the winning part." (point to the darker object)

- Pair BC: "In this video, the two objects come together to form a new object, and this feature of the object is the winning part." (point to the darker object)

- Pair CA: "And finally, in this video, the two objects come together to form a new object, and this feature of the object is the winning part." (point to the darker object)

\section{References}

Addis, D. R., Cheng, T., Roberts, R. P., \& Schacter, D. L. (2011). Hippocampal contributions to the episodic simulation of specific and general future events. Hippocampus, 21(10), 1045-1052. doi: 10.1002/hipo.20870

Ahmad, F. N., Fernandes, M., \& Hockley, W. E. (2015). Improving associative memory in older adults with unitization. Aging, Neuropsychology, and Cognition, 22(4), 452-472. doi:10.1080/ 13825585.2014.980216

Bader, R., Mecklinger, A., Hoppstadter, M., \& Meyer, P. (2010). Recognition memory for one-trial-unitized word pairs: Evidence from event-related potentials. NeuroImage, 50(2), 772-781.

Bastin, C., Diana, R. A., Simon, J., Collette, F., Yonelinas, A. P., \& Salmon, E. (2013). Associative memory in aging: The effect of unitization on source memory. Psychology \& Aging, 28(1), 275283.

Bower, G. H. (1970). Imagery as a relational organizer in associative learning. Journal of Verbal Learning and Verbal Behavior, 9(5), 529-533. doi:10.1016/S0022-5371(70)80096-2

Cermak, L.S. (1975). Imagery as an aid to retrieval for Korsakoff patients. Cortex, 11, 163-169

Cohen, N. J. (2015). Navigating life. Hippocampus, 25(6), 704-708. doi: 10.1002/hipo. 22443
D’Angelo, M. C., Kacollja, A., Rabin, J. S., Rosenbaum, R. S., \& Ryan, J. D. (2015). Unitization supports lasting performance and generalization on a relational memory task: Evidence from a previously undocumented developmental amnesic case. Neuropsychologia, 77, 185-200. doi:10.1016/j.neuropsychologia.2015.07.025

D’Angelo, M. C., Smith, V. M., Kacollja, A., Zhang, F., Binns, M. A., Barense, M. D., \& Ryan, J. D. (2016). The effectiveness of unitization in mitigating age-related relational learning impairments depends on existing cognitive status. Aging, Neuropsychology, and Cognition, 23(6), 667-690. doi:10.1080/13825585.2016.1158235

Delhaye, E., \& Bastin, C. (2016). The impact of aging on associative memory for preexisting unitized associations. Aging, Neuropsychology, and Cognition.. doi:10.1080/13825585.2016. 1263725

Driscoll, I., Hamilton, D. A., Petropoulos, H., Yeo, R. A., Brooks, W. M., Baumgartner, R. N., \& Sutherland, R. J. (2003). The aging hippocampus: Cognitive, biochemical and structural findings. Cerebral Cortex, 13(12), 1344-1351.

Duff, M. C., \& Brown-Schmidt, S. (2012). The hippocampus and the flexible use and processing of language. Frontiers in Human Neuroscience, 6, 69. doi:10.3389/fnhum.2012.00069

Eichenbaum, H., \& Cohen, N. J. (2001). From conditioning to conscious recollection: Memory systems of the brain. New York: Oxford University Press.

Ericsson, K. A., \& Kintsch, W. (1995). Long term working memory. Psychological Review, 102, 211-245.

Glisky, E. L., Schacter, D. L., \& Tulving, E. (1986). Learning and retention of computer-related vocabulary in memory-impaired patients: method of vanishing cues. Journal of Clinical and Experimental Neuropsychology, 8(3), 292-312. doi:10.1080/01688638608401320

Graf, P., \& Schacter, D. L. (1989). Unitization and grouping mediate dissociations in memory for new associations. Journal of Experimental Psychology: Learning, Memory, and Cognition, 15(5), 930.

Kan, I. P., Keane, M. M., Martin, E., Parks-Stamm, E. J., Lewis, L., \& Verfaellie, M. (2011). Implicit memory for novel associations between pictures: effects of stimulus unitization and aging. Memory \& Cognitition, 39, 778-790.

Kintsch, W. (1988). The role of knowledge in discourse comprehension: A construction integration model. Psychological Review, 95, 163182.

Konkel, A., Warren, D. E., Duff, M. C., Tranel, D. N., \& Cohen, N. J. (2008). Hippocampal amnesia impairs all manner of relational memory. Frontiers in Human Neuroscience, 2, 15. doi:10.3389/neuro.09. 015.2008

McGee, R. (1980). Imagery and recognition memory: The effects of relational organization. Memory and Cognition, 8(5), 394-399. doi:10.3758/BF03211135

Moscovitch, M. (2008). The hippocampus as a "stupid", domain-specific module: Implications for theories of recent and remote memory, and of imagination. Canadian Journal of Experimental Psychology, 62(1), 62-79. doi:10.1037/1196-1961.62.1.62

Moses, S. N., Ostreicher, M. L., Rosenbaum, R. S., \& Ryan, J. D. (2008). Successful transverse patterning in amnesia using semantic knowledge. Hippocampus, 18(2), 121-124. doi:10.1002/hipo.20378

Moses, S. N., Ostreicher, M. L., \& Ryan, J. D. (2010). Relational framework improves transitive inference across age groups. Psychological Research, 74(2), 207-218. doi:10.1007/s00426009-0244-0

Nasreddine, Z. S., Phillips, N. A., Bédirian, V., Charbonneau, S., Whitehead, V., Collin, I., ... \& Chertkow, H. (2005). The Montreal Cognitive Assessment, MoCA: A brief screening tool for mild cognitive impairment. Journal of the American Geriatrics Society, 53(4), 695-699. doi:10.1111/j.1532-5415.2005.53221.x

Naveh-Benjamin, M., Guez, J., Kilb, A., \& Reedy, S. (2004). The associative memory deficit of older adults: Further support using face- 
name associations. Psychology and Aging, 19(3), 541-546. doi:10. 1037/0882-7974.19.3.541

Old, S. R., \& Naveh-Benjamin, M. (2008). Differential effects of age on item and associative measures of memory: A meta-analysis. Psychology and Aging, 23(1), 104-118. doi:10.1037/0882-7974. 23.1.104

Ostreicher, M. L., Moses, S. N., Rosenbaum, R. S., \& Ryan, J. D. (2010). Prior experience supports new learning of relations in aging. The Journal of Gerontology. Psychological Sciences, 65B(1), 32-41. doi:10.1093/geronb/gbp081

Paivio, A. (1991). Dual coding theory: Retrospect and current status. Canadian Journal of Psychology, 45(3), 255-287.

Parks, C. M., \& Yonelinas, A. P. (2015). The importance of unitization for familiarity-based learning. Journal of Experimental Psychology: Learning, Memory, and Cognition, 41(3), 881-903.

Quamme, J. R., Yonelinas, A. P., \& Norman, K. A. (2007). Effect of unitization on associative recognition in amnesia. Hippocampus, 17(3), 192-200. doi:10.1002/hipo.20257

Rickard, T. C., \& Grafman, J. (1998). Losing their configural mind. Amnesic patients fail on transverse patterning. Journal of Cognitive Neuroscience, 10(4), 509-524.

Rickard, T. C., Verfaellie, M., \& Grafman, J. (2006). Transverse patterning and human amnesia. Journal of Cognitive Neuroscience, 18(10), 1723-1733. doi:10.1162/jocn.2006.18.10.1723
Ryan, J. D., Althoff, R. R., Whitlow, S., \& Cohen, N. J. (2000). Amnesia is a deficit in relational memory. Psychological Science, 11(6), 454 461.

Ryan, J. D., Leung, G., Turk-Browne, N. B., \& Hasher, L. (2007). Assessment of age-related changes in inhibition and binding using eye movement monitoring. Psychology and Aging, 22(2), 239-250. doi:10.1037/0882-7974.22.2.239

Ryan, J. D., Moses, S. N., Barense, M., \& Rosenbaum, R. S. (2013). Intact learning of new relations in amnesia as achieved through unitization. Journal of Neuroscience, 33(23), 9601-9613. doi:10. 1523/JNEUROSCI.0169-13.2013

Spence, K. W. (1952). The nature of the response in discrimination learning. Psychological Review, 59(1), 89-93.

Watson, H. C., Wilding, E. L., \& Graham, K. S. (2012). A role for perirhinal cortex in memory for novel object-context associations. Journal of Neuroscience, 32(13), 4473-4481.

Yonelinas. (1997). Recognition memory ROCs for item and associative information: The contribution of recollection and familiarity. Memory \& Cognition, 25(6), 747-763.

Zheng, Z., Li, J., Xiao, F., Ren, W., \& He, R. (2016). Unitization improves source memory in older adults: An event-related potential study. Neuropsychologia, 89, 232-244. 